\title{
Stability of Deterministic Finite State Machines
}

\author{
Danielle C. Tarraf ${ }^{1}$ \\ Munther A. Dahleh ${ }^{2}$ \\ Alexandre Megretski ${ }^{3}$
}

\begin{abstract}
We approach deterministic finite state machines from a systems point of view and we consider three notions of input/output stability: finite gain input/output stability, external stability and incremental input/output stability, as they apply to this class of systems. We propose linear programming based algorithms for verifying stability of a system, or lack thereof, in the finite gain input/output and the external sense. We show that for this class of systems, incremental input/output stability and external stability are equivalent notions, stronger than the notion of finite gain input/output stability.
\end{abstract}

\section{INTRODUCTION}

Our interest in deterministic finite state machines (DFM) stems from our ongoing attempts to use these systems as approximations of dynamical systems having finite input and output alphabets [8]. The ultimate goal of our research is to find a procedure that would enable us to generate, for a class of systems, nominal finite state machine models with guaranteed error bounds that are usable for robust controller synthesis [3]. The issue of stability comes up in several ways in the context of this approach. Take, for instance, Hankel model order reduction: the procedure is applicable to stable LTI systems, is guaranteed to generate stable reduced order systems, and provides a quantitative measure of the quality of the approximation in the form of induced gain bounds that are compatible with robustness analysis. Similarly for our problem, it makes sense to seek some notion of stability that is relevant to the question of existence of good finite state machine approximations, and to ask whether the approximate models should preserve this notion of stability. It also makes sense to look for meaningful gain conditions to quantify the approximation error and the sensitivity of our nominal model, and hence of the synthesized closed loop system, to perturbations due to unmodeled dynamics.

Various notions of stability have been explored for a range of discrete event and finite state systems. Traditionally, stability has been understood to mean that the system's state converges to some invariant subset of the states or visits a subset of the states infinitely often. In [6], logical discrete event systems were considered and two notions of stability, stability in the sense of Lyapunov and asymptotic stability, were defined; A Lyapunov function based approach was proposed for verifying stability of

\footnotetext{
1 Department of Mechanical Engineering, Massachusetts Institute of Technology,dtarraf@mit.edu

2 Department of Electrical Engineering and Computer Science, Massachusetts Institute of Technology, dahleh@mit.edu

3 Department of Electrical Engineering and Computer Science, Massachusetts Institute of Technology,ameg@mit.edu
}

logical discrete event systems with a metric state space. Questions of stability of equilibria and attractiveness of invariant sets were addressed for a class of autonomous hybrid automata in [2], where extensions of LaSalle's Invariance Theorem and Lyapunov's Indirect Method were proposed for nonblocking hybrid automata having certain properties. A notion of stability of periodic state trajectories for hybrid automata was considered in [4]. In [7], a necessary and sufficient condition for global stability of cyclic linear differential automata about a periodic trajectory is given. Notions of asymptotic stability and exponential stability of invariant sets for hybrid systems defined on an abstract time space were considered in [9], and sufficient conditions for stability were presented. An overview of results in Lyapunov stability of finite-dimensional hybrid systems was presented in [1], in particular as they pertain to switched linear systems. In [5], the problem of designing an output stabilizing controller for discrete-event dynamic systems was addressed, with the notion of output stability defined as the requirement that all state trajectories pass through a given set $E$ infinitely often.

Since our interest in deterministic finite state machines stems from problems of approximation and robust control, and keeping in mind that internal stability and input/output stability are generally decoupled notions in nonlinear systems, we focus in this paper on three potentially useful input/output notions of stability: The notion of finite gain input/output stability, which is widely used in classical LTI robust control. The notion of incremental input/output stability, which quantifies the sensitivity of the output of a system to perturbations in its input, and which is a possible alternative to the notion of finite gain input/output stability for robustness analysis of nonlinear systems. The notion of external stability, which captures the sensitivity of the output of a system to changes in its initial conditions, and which was introduced in [8] and shown to be of relevance to the problem of approximating an analog state system with finite input alphabet and output quantizer by a deterministic finite state machine.

\section{OVERVIEW OF THE PAPER}

\section{A. The Nominal System Model}

The nominal system models of interest to us are time driven, deterministic finite state machines. These models are understood to be approximations of discrete-time dynamical systems with finite input and output alphabets.

Definition 1: A deterministic finite state machine (DFM) $S$ is a set of finite alphabets $\{\mathcal{U}, \mathcal{Y}, \mathcal{Q}\}$ and a set of maps 
$\{f: \mathcal{Q} \times \mathcal{U} \rightarrow \mathcal{Q}, h: \mathcal{Q} \rightarrow \mathcal{Y}\}$.

$\mathcal{U}$ is a finite alphabet set of possible instantaneous values of the input. Thus input signal $u$ is understood to be an infinite string over alphabet set $\mathcal{U}$. Similarly, $\mathcal{Y}$ is a finite alphabet set of possible instantaneous values of the output. $\mathcal{Q}$ is a finite set of states of the machine. $f$ is the state transition function. $h$ is the output function, assumed, without lack of generality, to define a partition on $\mathcal{Q}$. The input and output alphabet sets, $\mathcal{U}$ and $\mathcal{Y}$, are assumed to be subsets of the set of integers containing zero. This assumption is not too restrictive, since an arbitrary finite set of symbols can always be bijectively mapped to a finite subset of the set of integers. What constitutes a meaningful way of defining such a mapping depends on the specifics of the particular problem: a primary issue is to identify a value of the input and a value of the output, associated with some nominal configuration of the system, to be mapped to the integer zero.

\section{B. The Scope of the Paper}

We consider deterministic finite machines, and we define three notions of input/output stability as they apply to this class of systems: finite gain input/output stability, external stability and incremental input/output stability. We present algorithms for verifying stability of a given machine $S$, or the lack thereof, for each of the notions considered. We also explore the relation between these three notions for the systems of interest: We show that the notions of external stability and incremental input/output stability are equivalent for this class of systems, and that input/output stability is a weaker notion.

\section{NOTIONS OF STABILITY}

We begin by precisely defining three notions of input/output stability as they apply to the systems of interest.

\section{A. Finite Gain Input/Output Stability}

Definition 2: A deterministic finite state machine $S$ is said to be finite gain input/output stable if there exists finite non-negative constants $C$ and $\gamma$ such that the inequality:

$$
\sum_{t=0}^{T}|y(t)| \leq C+\gamma \sum_{t=0}^{T}|u(t)|
$$

holds for all time $T \geq 0$, for all initial conditions of the system and for all admissible input sequences.

For a system that is input/output stable, the greatest lower bound of $\gamma$ is called the gain of $S$ and denoted by $\gamma_{o}$.

\section{B. External Stability}

Definition 3: A deterministic finite state machine $S$ is said to be externally stable if the following two conditions are satisfied:

(a) $S$ is finite gain input/output stable (b) There exists a finite time $\tau>0$ such that for every input $\{u(t)\}_{t=0}^{\infty}$ and for any two initial conditions of the system, $q_{1}(0)$ and $q_{2}(0)$, the corresponding outputs $\left\{y_{1}(t)\right\}_{t=0}^{\infty}$ and $\left\{y_{2}(t)\right\}_{t=0}^{\infty}$ satisfy $y_{1}(t)=$ $y_{2}(t)$ for all $t \geq \tau$

\section{Incremental Input/Output Stability}

For any set $\mathcal{A}$, define a function $d: \mathcal{A} \times \mathcal{A} \rightarrow\{0,1\}$ by the following rule:

$$
d(x, y) \doteq\left\{\begin{array}{cc}
0 & \text { if } x=y \\
1 & \text { otherwise }
\end{array}\right.
$$

Function $d$ defines a metric that induces the discrete topology on set $\mathcal{A}$.

Definition 4: A deterministic finite state machine $S$ is said to be incrementally input/output stable if the following two conditions are satisfied:

(a) There exists a finite time $\hat{\tau}>0$ such that the output of $S$ corresponding to an all zero input satisfies $y(t)=0$ for $t \geq \hat{\tau}$, for any initial condition

(b) There exists finite, non-negative constants $\hat{C}$ and $\hat{\gamma}$ such that for any two pairs of input sequence and initial state, $\left(\left\{u_{1}(t)\right\}_{t=0}^{\infty}, q_{1}(0)\right)$ and $\left(\left\{u_{2}(t)\right\}_{t=0}^{\infty}, q_{2}(0)\right)$, the corresponding outputs, $\left\{y_{1}(t)\right\}_{t=0}^{\infty}$ and $\left\{y_{2}(t)\right\}_{t=0}^{\infty}$, satisfy the inequality:

$$
\sum_{t=0}^{T} d\left(y_{1}(t), y_{2}(t)\right) \leq \hat{C}+\hat{\gamma} \sum_{t=0}^{T} d\left(u_{1}(t), u_{2}(t)\right)
$$

for all time $T \geq 0$.

For a system that is incrementally input/output stable, the greatest lower bound of $\hat{\gamma}$ is called the incremental gain of $S$ and denoted by $\hat{\gamma}_{o}$.

\section{VERIFYING STABILITY}

\section{A. Verifying Finite Gain Input/Output Stability}

Lemma 1: Let $S$ be a deterministic finite state machine. The following two statements are equivalent:

(a) $S$ is finite gain input/output stable

(b) There exists a finite time $\tau>0$ such that the output of $S$ corresponding to an all zero input satisfies $y(t)=0$ when $t \geq \tau$ for any initial condition

Proof: First, we prove the statement (a) $\Rightarrow$ (b): When $S$ has finite gain and $u(t)=0$ for all time $t$, the gain condition eq (1) reduces to $\sum_{t=0}^{T}|y(t)| \leq C$ for all $T \geq 0$. But $C$ is finite and $y$ takes its values in a subset of the set of integers. Hence there must exist some finite time $\tau$ such that $y(t)=0$ for all $t \geq \tau$.

To prove the statement (b) $\Rightarrow$ (a), suppose that $S$ is not finite gain input/output stable. Then there must exist at least one 
feasible state trajectory, beginning and ending at the same state, and such that the cumulative input that drives the system once along this cyclic trajectory is exactly zero while the cumulative output of the system as its state evolves once along this cyclic trajectory is strictly positive. For if that were not the case, then a sufficiently large but finite choice of $C$ and $\gamma$ would guarantee that the gain condition, eq (1), holds. (For instance, $C=\gamma=n l$ where $n=\operatorname{card}(\mathcal{Q})$ and $l=\max _{y \in \mathcal{Y}}|y|$ would be one such possible choice). Therefore, the response of $S$ to an all zero input string when the system is initialized to any of the states of this cycle can never settle to zero.

Lemma 1 seems to be a negative statement in the sense that for the systems of interest, finiteness of the gain does not tell us very much about the input/output behavior of the system beyond its 'autonomous' behavior, and even less about the sensitivity of the system to perturbations. It remains to be seen whether this notion of stability can be of use within a robust analysis and control framework.

Intuitively, Lemma 1 says that $S$ is finite gain input/output stable iff all its zero input state trajectories converge to an invariant subset of $\mathcal{Q}$ that only contains states whose corresponding output is zero. The next result builds on this intuition.

Let $\mathcal{Q}_{o}=\{q \in \mathcal{Q} \mid h(q)=0\}$. Define an indicator function $\theta: \mathcal{Q} \rightarrow\{0,1\}$ by the following rule:

$$
\theta(q) \doteq\left\{\begin{array}{cc}
0 & q \in \mathcal{Q}_{o} \\
1 & \text { otherwise }
\end{array}\right.
$$

Theorem 1: $S$ is finite gain input/output stable if and only if there exists a non-negative function of the state, $V: \mathcal{Q} \rightarrow \mathbf{R}^{+}$such that eq (5) holds for all $q_{i} \in \mathcal{Q}$ :

$$
V\left(f\left(q_{i}, 0\right)\right)-V\left(q_{i}\right) \leq-\theta\left(q_{i}\right)
$$

Proof: To prove sufficiency, note that when $u(t)=0$ for all $t \geq 0$, eq (5) implies that:

$$
V(q(t+1))-V(q(t)) \leq-\theta(q(t))
$$

holds for all $t \geq 0$. Hence for any initial condition $q(0)$, and for any time $T>0$, by summing up eq (6) along the state trajectory from $t=0$ to $t=T$, we get:

$$
V(q(T+1))-V(q(0)) \leq-\sum_{t=0}^{T} \theta(q(t))
$$

Hence, we have:

$$
V(q(0)) \geq \sum_{t=0}^{T} \theta(q(t))
$$

There must exist some finite time $\tau$ such that $\theta(q(t))=0$ for $t \geq \tau$, otherwise the summation on the right hand side of eq (8) can be made infinite and hence $V(q(0))$ would be infinite as well, which is not the case when the linear program is feasible. Hence $y(t)=h(q(t))=0$ for all $t \geq \tau$ and $S$ is finite gain input/output stable by Lemma 1 .
To prove necessity we assume $S$ is finite gain input/output stable and we explicitly show how to construct a function $V$ that satisfies eq (5). Define function $V: \mathcal{Q} \rightarrow \mathbf{R}^{+}$by the following rule:

$$
V\left(q_{i}\right)=\sum_{t=0}^{\infty}\left|y_{i}(t)\right|
$$

where $\left\{y_{i}(t)\right\}$ is the output trajectory corresponding to initial state $q_{i}$ and an all zero input string. Since $S$ is finite gain input/output stable, by Lemma 1 there exists a time $\tau$ such that $y_{i}(t)=0$ for $t \geq \tau$. Hence, function $V$ is bounded and non-negative by construction. Moreover, we have:

$$
V\left(f\left(q_{i}, 0\right)\right)-V\left(q_{i}\right)=\sum_{t=0}^{\tau}\left|y_{k}(t)\right|-\sum_{t=0}^{\tau}\left|y_{i}(t)\right|
$$

where $\left\{y_{k}(t)\right\}$ is the output trajectory corresponding to initial state $q_{k}=f\left(q_{i}, 0\right)$ and an all zero input string. But, for any $i$, we have:

$$
\sum_{t=0}^{\tau}\left|y_{i}(t)\right|=\sum_{t=0}^{\tau}\left|y_{k}(t)\right|+\left|h\left(q_{i}\right)\right|
$$

and also:

$$
\left|h\left(q_{i}\right)\right| \geq \theta\left(q_{i}\right)
$$

Hence, the inequality $V\left(f\left(q_{i}, 0\right)\right)-V\left(q_{i}\right) \leq-\theta\left(q_{i}\right)$ holds for every $q_{i} \in \mathcal{Q}$.

Therefore, the problem of verifying finite gain input/output stability of a given finite state machine with $n$ states is equivalent to checking feasibility of a linear program of the form $\mathbf{A x} \geq \mathbf{b}, \mathbf{x} \geq \mathbf{0}$, where the components of $\mathbf{x}$, the $n$-dimensional vector of decision variables, correspond to the values of the function $V$ at each point in $\mathcal{Q}$. Matrix A is an $n \times n$ matrix of 0's, 1's and -1 's. Each row of $\mathbf{A}$ is either identically zero or consists of a " 1 " entry along the diagonal and a "-1" elsewhere. Vector $\mathbf{b}$ is an $n$-dimensional vector of 0 's and 1 's.

Once the finite gain input/output stability of a DFM has been established, its gain can be computed, if desired, using a bisection algorithm based on Theorem 1 in [3].

\section{B. Verifying External Stability}

Let $\hat{\mathcal{Q}}_{o}=\left\{\left(q_{1}, q_{2}\right) \in \mathcal{Q}^{2} \mid h\left(q_{1}\right)=h\left(q_{2}\right)\right\}$. Define an indicator function $\phi: \mathcal{Q}^{2} \rightarrow\{0,1\}$ by the following rule:

$$
\phi\left(q_{1}, q_{2}\right) \doteq\left\{\begin{array}{cc}
0 & \left(q_{1}, q_{2}\right) \in \hat{\mathcal{Q}}_{o} \\
1 & \text { otherwise }
\end{array}\right.
$$

Theorem 2: $S$ is externally stable if and only if it is finite gain input/output stable and there exists a non-negative function $V: \mathcal{Q}^{2} \rightarrow \mathbf{R}^{+}$such that eq (14) holds for all pairs of states $\left(q_{1}, q_{2}\right) \in \mathcal{Q}^{2}$ and for all $u \in \mathcal{U}$.

$$
V\left(\left(f\left(q_{1}, u\right), f\left(q_{2}, u\right)\right)\right)-V\left(\left(q_{1}, q_{2}\right)\right) \leq-\phi\left(q_{1}, q_{2}\right)
$$

Proof: To prove sufficiency, suppose that $S$ is finite gain input/output stable and that function $V$ with the desired 
properties exists. Note that for any input $\{u(t)\}_{t=0}^{\infty}$ and for any two initial conditions $q_{1}(0)$ and $q_{2}(0)$, eq (14) implies the following inequality holds for all segments of the corresponding state trajectories $\left\{q_{1}(t)\right\}_{t=0}^{\infty}$ and $\left\{q_{2}(t)\right\}_{t=0}^{\infty}$ :

$$
\begin{gathered}
V\left(f\left(q_{1}(t), u(t)\right), f\left(q_{2}(t), u(t)\right)\right)-V\left(q_{1}(t), q_{2}(t)\right) \\
\leq-\phi\left(q_{1}(t), q_{2}(t)\right)
\end{gathered}
$$

Eq (15) is satisfied for all $t \geq 0$. Hence, by summing up eq (15) from $t=0$ to $t=T$, we obtain:

$$
\begin{gathered}
V\left(f\left(q_{1}(T), u(T)\right), f\left(q_{2}(T), u(T)\right)\right)-V\left(q_{1}(0), q_{2}(0)\right) \\
\leq-\sum_{t=0}^{T} \phi\left(q_{1}(t), q_{2}(t)\right)
\end{gathered}
$$

Thus, for any $T>0$ we have:

$$
V\left(q_{1}(0), q_{2}(0)\right) \geq \sum_{t=0}^{T} \phi\left(q_{1}(t), q_{2}(t)\right)
$$

Since the linear program is assumed to be feasible, $V\left(q_{1}(0), q_{2}(0)\right)$ is bounded. Hence, for every input sequence $\{u(t)\}$, there must exist some finite time $\tau>0$ such that $\phi\left(q_{1}(t), q_{2}(t)\right)=0$ for all $t \geq \tau$. Moreover, the smallest such time $\tau$ is bounded above by $n^{2}$, where $n=\operatorname{card}(\mathcal{Q})$. For if that were not the case, we can construct an input sequence for which no such $\tau$ exists, leading to a contradiction. Therefore, there exists a finite time $\tau>0$ such that for any pair of initial conditions $\left(q_{1}(0), q_{2}(0)\right)$, and for any input sequence $\{u(t)\}_{t=0}^{\infty}$, the corresponding output sequences match starting at time $\tau$ : $y_{1}(t)=y_{2}(t)$ for all $t \geq \tau$.

To prove necessity, assume that $S$ is externally stable. Thus there exists a finite time $\tau>0$ such that for any input sequence $\{u(t)\}_{t=0}^{\infty}$ and for any pair of initial conditions, $\left(q_{1}, q_{2}\right) \in \mathcal{Q}^{2}$, the corresponding output sequences $\left\{y_{1}(t)\right\}_{t=0}^{\infty}$ and $\left\{y_{2}(t)\right\}_{t=0}^{\infty}$ satisfy $y_{1}(t)=y_{2}(t)$ for all $t \geq \tau$, or equivalently $d\left(y_{1}(t), y_{2}(t)\right)=0$ for all $t \geq \tau$. Define function $V: \mathcal{Q}^{2} \rightarrow \mathbf{R}^{+}$by the following rule:

$$
\begin{gathered}
V\left(q_{1}, q_{2}\right) \doteq \max _{u \in \mathcal{U}^{\infty}} \sum_{t=0}^{\infty} d\left(y_{1}(t), y_{2}(t)\right) \\
\doteq \max _{u \in \mathcal{U}^{\tau}} \sum_{t=0}^{\tau} d\left(y_{1}(t), y_{2}(t)\right)
\end{gathered}
$$

$V$ is bounded and non-negative by construction. To show that $V$ satisfies eq (14), note that for any pair $\left(q_{1}, q_{2}\right) \in \mathcal{Q}^{2}$ and for any input $u \in \mathcal{U}$ we have:

$$
V\left(q_{1}, q_{2}\right) \geq V\left(f\left(q_{1}, u\right), f\left(q_{2}, u\right)\right)+\phi\left(q_{1}, q_{2}\right)
$$

Equality is achieved when an optimal pair of state trajectories $\left(\left\{q_{1}^{o}(t)\right\},\left\{q_{2}^{o}(t)\right\}\right)$, corresponding to initial states $q_{1}^{o}(0)=q_{1}$ and $q_{2}^{o}(0)=q_{2}$ and whose corresponding output trajectories $\left(\left\{y_{1}^{o}(t)\right\},\left\{y_{2}^{o}(t)\right\}\right)$ define the value of $V\left(q_{1}, q_{2}\right)$, are such that $q_{1}^{o}(1)=f\left(q_{1}, u\right)$ and $q_{2}^{o}(1)=f\left(q_{2}, u\right)$.
Therefore, the problem of verifying that a finite gain input/output stable DFM system $S$ with $n$ states is externally stable is equivalent to the problem of checking feasibility of a linear program of the form $\mathbf{A x} \geq \mathbf{b}, \mathbf{x} \geq \mathbf{0}$. The components of $\mathbf{x}$, the $n^{2}$-dimensional vector of decision variables, correspond to the values of the function $V$ at each point in $\mathcal{Q}^{2}$. Matrix A consists of 0's, 1's and -1's and has dimension $a n^{2} \times n^{2}$, where $a$ is the cardinality of the input alphabet set $\mathcal{U}$. The entries along each row of $\mathbf{A}$ sum up to zero and include at most two non-zero values. Vector $\mathbf{b}$ is an $n^{2}$-dimensional vector of 0's and 1's.

It will be shown in the next section that the problem of verifying incremental input/output stability for this class of systems is equivalent to the problem of verifying external stability.

\section{RELATION BETWEEN NOTIONS OF STABILITY}

In this section, we explore the relation between the three notions of input/output stability stability considered for deterministic finite state machines.

Theorem 3: Let $S$ be a finite state machine and consider the following three statements:

(a) $\quad S$ is incrementally input/output stable

(b) $S$ is externally stable

(c) $S$ is finite gain input/output stable

The statement (a) $\Leftrightarrow$ (b) $\Rightarrow$ (c) is true. The statement (c) $\Rightarrow(\mathrm{b})$ is not true.

Proof: The implication (b) $\Rightarrow$ (c) follows immediately from Definition 3.

To show that $(\mathrm{a}) \Rightarrow(\mathrm{b})$, note that when $u_{1}(t)=u_{2}(t)$ for all $t$, eq (3) reduces to $\sum_{t=0}^{T} d\left(y_{1}(t), y_{2}(t)\right) \leq \hat{C}$, for all $T \geq 0$. Since $\hat{C}$ is finite and function $d$ takes on values in $\{0,1\}$, there exists some finite $\tau$ such that $d\left(y_{1}(t), y_{2}(t)\right)=$ 0 for all $t \geq \tau$, regardless of initial conditions. Moreover, it follows from Definition 4 and Lemma 1 that $S$ is finite gain input/output stable. Hence, $S$ is externally stable.

To show that (b) $\Rightarrow$ (a), suppose that $S$ is not incrementally input/output stable. Then there exists a pair of initial states $\left(q_{1}^{o}, q_{2}^{o}\right)$ and a pair of inputs $\left(\left\{u_{1}(t)\right\},\left\{u_{2}(t)\right\}\right)$ that differ by only a finite number of terms, and whose corresponding outputs $\left\{y_{1}(t)\right\}$ and $\left\{y_{2}(t)\right\}$ differ by an infinite number of terms. For this choice of initial states and inputs, let $t_{o} \geq 0$ be the smallest time such that: $u_{1}(t)=u_{2}(t)$ for $t \geq t_{o}$. The corresponding outputs $\left\{y_{1}(t)\right\}$ and $\left\{y_{2}(t)\right\}$ satisfy the condition that for every $t \geq t_{o}$, there exists some $\tau \geq t$ such that $y_{1}(\tau) \neq y_{2}(\tau)$. Now consider input $\{u(t)\}$ defined as follows:

$$
u(t) \doteq u_{1}\left(t+t_{o}\right)
$$

Set $q_{1}(0)$ equal to the state of the machine $S$ at time $t_{o}$ when $S$ is initialized to $q_{1}^{o}$ and driven by input $u_{1}$. Similarly, set $q_{2}(0)$ equal to the state of the machine $S$ at time $t_{o}$ when $S$ is initialized to $q_{2}^{o}$ and driven by input $u_{2}$. Note that by construction, there cannot exist a time $\tau$ 


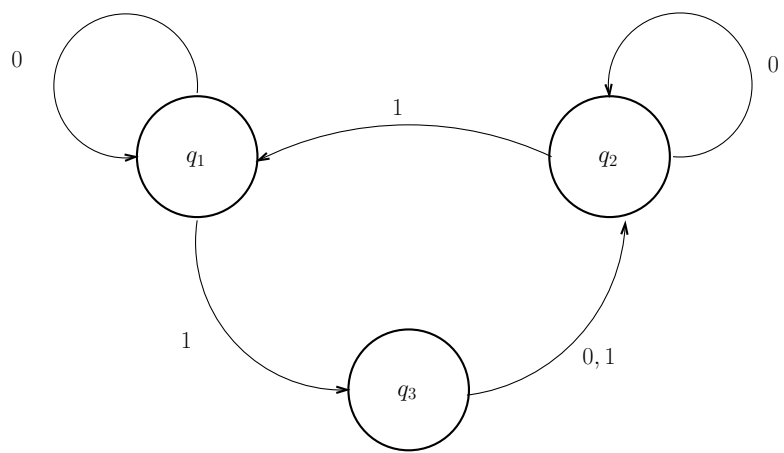

Fig. 1. Counterexample 1: State transition diagram

such that the outputs of $S$ corresponding to $\left(\{u(t)\}, q_{1}(0)\right)$ and $\left(\{u(t)\}, q_{2}(0)\right)$ become equal for all $t \geq \tau$. Hence $S$ is not externally stable.

To show that the statement (c) $\Rightarrow$ (b) does not hold, we give the following counterexample.

Counterexample 1: Consider a finite state machine $S$ with 3 states, $\mathcal{Q}=\left\{q_{1}, q_{2}, q_{3}\right\}$, with binary input and output alphabet sets $\mathcal{U}=\mathcal{Y}=\{0,1\}$ and with transition function $f$ defined by the rule:

$$
f \doteq\left\{\begin{array}{l}
f\left(q_{1}, 0\right)=q_{1} \\
f\left(q_{1}, 1\right)=q_{3} \\
f\left(q_{2}, 0\right)=q_{2} \\
f\left(q_{2}, 1\right)=q_{1} \\
f\left(q_{3}, 0\right)=q_{2} \\
f\left(q_{3}, 1\right)=q_{2}
\end{array}\right.
$$

The state transition diagram of this machine is given in Figure 1. The output function $h$ is defined by the rule:

$$
h \doteq\left\{\begin{array}{l}
h\left(q_{1}\right)=0 \\
h\left(q_{2}\right)=0 \\
h\left(q_{3}\right)=1
\end{array}\right.
$$

By inspection, it is easy to see that this system is finite gain input/output stable since the only zero-input cyclic trajectories are the self transitions of $q_{1}$ and $q_{2}$, both states for which the output is zero. To check this formally, note that function $V$ taking the value 0 at $q_{1}$ and $q_{2}$ and taking the value 1 at $q_{3}$ is a feasible solution to the linear program proposed in Theorem 1.

However, this system is clearly not externally stable; When the initial state is $q(0)=q_{1}$ and the input is constantly 1 , the output is $0100100100 \ldots$... On the other hand, when the initial state is $q(0)=q_{3}$ and for the same input as before, the output is $1001001001 \ldots$... By inspection, the outputs cannot become equal from some time $\tau$ and on.

Remark 1: The equivalence between the notions of external stability and incremental input/output stability is specific to deterministic finite state machines. We do not expect this result to carry through in the general setting for arbitrary nonlinear systems.

\section{CONCLUSIONS AND FUTURE WORK}

We considered deterministic finite state machine systems and defined three notions of input/output stability as they apply to this class of systems: finite gain input/output stability, external stability and incremental input/output stability. We proposed linear programming based algorithms to verify stability in the finite gain input/output sense and the external sense. We showed that the notions of incremental input/output stability and of external stability are equivalent for this class of systems. We also showed that they are much stronger than the notion of input/output stability for this class of systems. This is not a surprising result since the systems in question are not linear, and it is well known that nonlinear systems can have small gain and infinite incremental gain.

On the negative side, the problem of external stability verification may not be practically computable for many machines of interest (based on our results, in order to verify external stability of a machine of size $n$, we need to check feasibility of a linear program with $n^{2}$ decision variables). Future work will focus on constructing more efficient algorithms for external stability verification and computation of incremental gain bounds. Moreover, it is yet to be shown that these notions of stability can be used to express practical control objectives or to formulate robustness criteria, as in the case of classical robust control. Thus, another research direction is exploring the utility of these three notions in a robust analysis or control framework.

\section{REFERENCES}

[1] R. DeCarlo, M. Branicky, S. Pettersson and B. Lennarston, "Perspectives and results on the stability and stabilizability of hybrid systems", Proceedings of the IEEE, vol. 88, no. 7, pp.1069-1082, July 2000.

[2] J. Lygeros, K. Johansson, S. Simic, Z. Jun and S. Sastry, "Dynamical properties of hybrid automata", IEEE Transactions on Automatic Control, vol. 48, no. 1, pp. 2-17, January 2003.

[3] A. Megretski, "Robustness of finite state automata", in Proceedings of the Mohammed Dahleh Memorial Symposium, Santa Barbara, CA, February 2002.

[4] M. Mohrenschildt, "Hybrid systems: Solutions, stability, control", Proceedings of the American Control Conference, pp.692-698, June 2000.

[5] C. Ozveren and A. Willsky, "Output stabilizability of discrete-event dynamic systems", IEEE Transactions on Automatic Control, vol. 36, no.8, pp. 925-935, August 1991.

[6] K. Passino, A. Michel and P. Antsaklis, "Lyapunov stability of a class of discrete event systems", IEEE Transactions on Automatic Control, vol. 39, no. 2, pp. 269-279, February 1994.

[7] A. Savkin and A. Matveev, "Qualitative analysis of differential automata: Existence and stability of limit cycles", in Information, Decision and Control, pp.265-270, February 1999.

[8] D. Tarraf, A. Megretski and M.A. Dahleh, "Finite automata approximations with error bounds for systems with quantized actuation and measurement: A case study", Proceedings of the 43rd IEEE Conference on Decision and Control, pp.1436-1441, December 2004.

[9] H. Ye, A. Michel and L. Hou, "Stability theory for hybrid dynamical systems", IEEE Transactions on Automatic Control, vol.43, pp.461474, April 1998. 